\title{
Hipertensão pulmonar associada à síndrome da imunodeficiência adquirida: apresentação de cinco casos e revisão da literatura
}

\section{Pulmonary hypertension associated with acquired immunodeficiency syndrome: presentation of five cases and review of the literature}

\author{
Adriano Assis Mendes ${ }^{1}$, Carlos Guilhermo Piscoya Roncal ${ }^{1}$, Vicente Luiz Vaz da Costa ${ }^{2}$, \\ Flávio Adolfo Aranha Japyassu ${ }^{3}$, Flávio Roberto Azevedo Oliveira ${ }^{3}$, \\ Diana Lamprea Sepúlveda ${ }^{1}$, Cícero Antônio da Silva ${ }^{1}$, Eugênio Soares Albuquerque ${ }^{1}$, \\ Roberto José Vieira de Melo ${ }^{4}$, Ricardo Loureiro ${ }^{5}$ Maria Tereza Cartaxo Muniz ${ }^{6}$
}

\begin{abstract}
RESUMO
Diversas doenças cardiorespiratórias podem complicar a síndrome da imunodeficiência adquirida. A hipertensão pulmonar é uma rara doença com um pobre prognóstico. Nós descrevemos esta síndrome em cinco pacientes com infecção pelo vírus da imunodeficiência adquirida em nosso serviço com revisão da literatura.
\end{abstract}

Palavras-chaves: Hipertensão pulmonar. Síndrome da imunodeficiência adquirida. Insuficiência cardíaca.

\section{ABSTRACT}

Several cardiorespiratory diseases may complicate the acquired immunodeficiency syndrome. Pulmonary hypertension is a rare clinical disorder with a poor prognosis. We describe this syndrome in five patients seen at our service who presented infection with the acquired immunodeficiency virus, and we review the literature.

Key-words: Pulmonary hypertension. Acquired immunodeficiency syndrome. Heart failure.

Alterações cardiovasculares relacionadas à síndrome de imunodeficiência adquirida (SIDA) são bem conhecidas, e incluem pericardite, miocardite e miocardiopatia dilatada. Atualmente com 0 aumento da sobrevida dos pacientes decorrente do tratamento antiretroviral (TARV) e profilaxia otimizada das infecções oportunistas, condições não infecciosas têm sido reconhecidas, entre elas a hipertensão arterial pulmonar (HAP). A hipertensão arterial pulmonar é uma doença grave da circulação pulmonar, caracterizada por alterações na parede vascular localizadas inicialmente nos segmentos pré-capilares da circulação pulmonar

1. Divisão de Cardiologia do Pronto Socorro de Cardiologia de Pernambuco, Universidade de Pernambuco, Recife; 2. Divisão de Doenças Infecciosas do Hospital Universitário Oswaldo Cruz, Universidade de Pernambuco, Recife. 3. Setor de Hemodinâmica, Hospital Memorial São José, Recife,PE. 4. Departamento de Patologia, Universidade Federal de Pernambuco, Recife, PE. 5. Setor de Imagem Cardiovascular CardioScan-Unineuro, Recife, PE. 6. Instituto de Ciências Biológicas, Universidade de Pernambuco, Recife, PE

Endereço para correspondência: Dr. Adriano Assis Mendes. Rua Neto de Mendonça, 165/904, Tamarineira, 52050-100 Recife, PE.

Tel 5581 9949-8200

e-mail: adrianoamendes@terra.com.br

Recebido para publicação em 19/02/2009

Aceito em 20/07/2009 e que evoluem com remodelação, vasoconstrição e trombose in situ..$^{31}$ Evolui com aumento progressivo da resistência vascular pulmonar, falência ventricular direita e morte quando não tratada. A incidência de HAP associada ao vírus da imunodeficiência adquirida (HIV) é $0,5 \%$, e na população geral em torno de $0,02 \%{ }^{15}$. Em estudo multicêntrico e prospectivo na era da TARV, a prevalência de HAP foi de $0,46 \%{ }^{33}$. Descrevemos as características clínicas, hemodinâmicas e evolução de cinco pacientes com HAP associadas ao HIV acompanhados na Divisão de Doenças Infecciosas e Pronto Socorro Cardiológico de Pernambuco (PROCAPE) do Hospital Universitário Oswaldo Cruz.

\section{RELATO DOS CASOS}

Foram analisados e acompanhados cinco pacientes com HAP associada ao HIV no período de 2005 a 2008 através de revisão de prontuários. Todos os casos de HIV relacionados a HAP estão sumarizados na Tabela 1 e 2. Dos cinco casos três eram do sexo feminino, a idade variou de 22 a 51 anos com média de 33 anos. A média do intervalo de tempo entre o diagnóstico de HIV e o diagnóstico de hipertensão pulmonar foi de 4,3 anos (variando de 3 meses a 13 anos). Não foi encontrada nenhuma relação 
TABELA 1

Características clínicas e hemodinâmicas de cinco pacientes com hipertensão pulmonar associada ao HIV.

\begin{tabular}{lrrrrrr}
\hline & Caso 1 & Caso 2 & Caso 3 & Caso 4 & Caso 5 & Média \\
\hline Sexo & F & M & M & F & F & F \\
Idade (anos) & 31 & 51 & 33 & 31 & 22 & 33 \\
CF(NYHA) & IV & III & II & IV & IV & - \\
PSAP (mmHg) & 91 & 110 & 115 & 97 & 108 & 104 \\
NT-proBNP(ng/mL) & 4028 & 9787 & 3773 & 1008 & 167 & 3752 \\
$\quad$ PmAP (mmHg) & 37 & 73 & 66 & 69 & 73 & 63 \\
$\quad$ PmAD & 9 & 31 & 27 & 15 & 36 & 23 \\
RPT(Dyn/seg/cm5) & 1148 & 4514 & 2632 & 2599 & 2132 & 2605 \\
RAP(Dyn/seg/cm5) & 775 & 3091 & 1914 & 2071 & 1606 & 1891 \\
IC(L/min/m²) & 1,85 & 0,79 & 1,29 & 1,47 & 1,98 & 1,47 \\
\hline
\end{tabular}

HIV: human immunodeficiency virus, CF: classe funcional, NYHA: New York Heart Association, PSAP: pressão sistólica artéria pulmonar, NT-proBNP: peptídeo natriurético cerebral, PmAP: pressão média artéria pulmonar, PmAD: pressão média átrio direito, RPT: resistência pulmonar total, RAP: resistência arteriolar pulmonar, IC: índice cardíaco.

TABELA 2

Tempo do diagnóstico de hipertensão pulmonar após diagnóstico da infecção pelo HIV, diagnóstico clínico e evolução.

\begin{tabular}{lccccc}
\hline & Caso 1 & Caso 2 & Caso 3 & Caso 4 & Caso 5 \\
\hline Diagnóstico HIV para HAP & 3 meses & 10 meses & 13 anos & 1,6 anos & 6anos \\
\hline Sintomas HAP até o & & & & & \\
diagnóstico de HAP & 6 meses & 6 meses & 3 meses & 5 meses & 4 meses \\
\hline Evolução HAP & óbito & óbito & óbito & viva & viva \\
\hline Após diagnóstico & 9 meses & 12 meses & 10 meses & 38 meses & 7 meses \\
\hline
\end{tabular}

HIV: human immunodeficiency virus, HAP: hipertensão arterial pulmonar.

existente entre contagens de células $\mathrm{CD} 4 \mathrm{e}$ carga viral ou presença de infecção com o desenvolvimento e progressão da HAP. Todos os pacientes estavam em uso de TARV.

Três dos cinco pacientes encontravam-se no momento do diagnóstico em classe funcional (CF) IV, de acordo com a New York Heart Association (NYHA) ${ }^{10}$. 0 intervalo de tempo médio entre os sintomas e o diagnóstico da hipertensão pulmonar foi de 4,8 meses ( 3 a 6 meses). 0 sintoma clínico mais importante foi a dispnéia, em 100\% dos casos (Tabela 3). Edema de membros inferiores esteve presente em todos os pacientes. Dor precordial apenas no caso 5. Outros sintomas referidos foram, cianose em três casos, fadiga em cinco, síncope em três casos. Os sinais principais do exame cardiológico foram os seguintes: segunda bulha (P2) hiperfonética, galope do ventrículo direito e sopro sistólico de regurgitação pulmonar esteviveram presentes em todos os pacientes no momento do diagnóstico. Outros sinais incluíram estase jugular importante, edema periférico, ascite e estertores pulmonares.

A radiografia de tórax demonstrou cardiomegalia e dilatação do tronco da artéria pulmonar em todos os pacientes. 0 eletrocardiograma (ECG) mostrou sinais de hipertrofia ventricular direita, desvio do eixo para direita, taquicardia sinusal e alterações de repolarização ventricular inespecíficas. Todos com ritmo sinusal. 0 ecocardiograma demonstrou em todos,
TABELA 3

Parâmetros clínicos de cinco pacientes com HAP associado ao HIV.

\begin{tabular}{lccccc}
\hline & Caso 1 & Caso 2 & Caso 3 & Caso 4 & Caso 5 \\
\hline Parâmetros clínicos & + & + & + & + & + \\
Dispnéia & + & + & + & + & + \\
Edema & + & + & + & + & + \\
Fadiga & + & + & + & + & + \\
P2 hiper & + & + & + & + & + \\
Galope de VD & + & + & + & + & + \\
Sopro IP & + & + & + & + & + \\
Estase jugular & + & + & + & + & + \\
Hepatomegalia & + & + & + & + & - \\
Síncope & + & + & - & - & + \\
Cianose & + & + & - & - & + \\
Ascite & + & + & - & + & - \\
Estertores pulmão & + & + & + & - & - \\
Dor precordial & - & - & - & - & + \\
\hline
\end{tabular}

HAP: hipertensão arterial pulmonar, HIV: human immunodeficiency virus, VD: ventrículo direito, IP: insuficiência pulmonar.

sinais de dilatação de câmaras direitas, regurgitação tricúspide e movimentação paradoxal do septo interventricular (SIV) (Figura 1A). A ressonância magnética de coração, mostrou dilatação importante do ventrículo direito com hipocontratilidade, hipertrofia de suas paredes, leve derrame pericárdico alem de rechaço importante do septo interventricular para 0 ventrículo esquerdo (Figura 1B). A pressão sistólica da artéria pulmonar (PSAP) foi mensurada através do refluxo tricúspide em todos os pacientes, variando de 91 a $115 \mathrm{mmHg}$ (média de $104 \mathrm{mmHg}$ ). 0 teste de caminhada dos seis minutos (TC6m) teve como média 156 metros. 0 peptídeo natriurético cerebral (NT-proBNP) foi mensurado em todos os pacientes com média de $3752 \mathrm{ng} / \mathrm{mL}$ (167 a $9787 \mathrm{ng} / \mathrm{mL})$.

0 diagnóstico de HAP foi confirmado pelo cateterismo cardíaco direito e esquerdo com teste de vasoreatividade pulmonar com óxido nítrico. A média da pressão média da artéria pulmonar foi de $63 \mathrm{mmHg}(37 \mathrm{a} 73 \mathrm{mmHg})$, pressão média do átrio direito $23 \mathrm{mmHg}$ ( 9 a $36 \mathrm{mmHg}$ ), resistência pulmonar total de 2605Dyn $/ \mathrm{seg} / \mathrm{cm}^{5}$ (1148 a 4514), resistência arteriolar pulmonar de 1891Dyn $/ \mathrm{seg} / \mathrm{cm}^{5}$ (775 a 3091), com índice cardíaco de 1,47L/ $\mathrm{min} / \mathrm{m}^{2}\left(0,79\right.$ a $\left.2,72 \mathrm{~L} / \mathrm{min} / \mathrm{m}^{2}\right)$.

As drogas utilizadas para o tratamento da HAP, relacionada ao HIV, incluíram sildenafil, bosentana, digital, furosemida, aldactone, oxigênio, warfarin. Apenas dois, caso 4 e 5, pacientes permanecem vivos, em uso de sildenafil associado ao bosentana há 38 meses e bosentana há 7 meses respectivamente. Os demais pacientes faleceram por insuficiência cardíaca direita refratária, sendo um (caso 2) por morte súbita.

0 estudo anatomo patológico realizado apenas no caso dois (Figuras 2A, 2B, 3A e 3B), demonstrou coração com significativo aumento de volume às custas do ventrículo direito, com dilatação da câmara ventricular associada a hipertrofia da parede. Os pulmões estavam congestos. Os grandes vasos, artéria pulmonar e aorta, apresentavam placas ateromatosas, assim como 


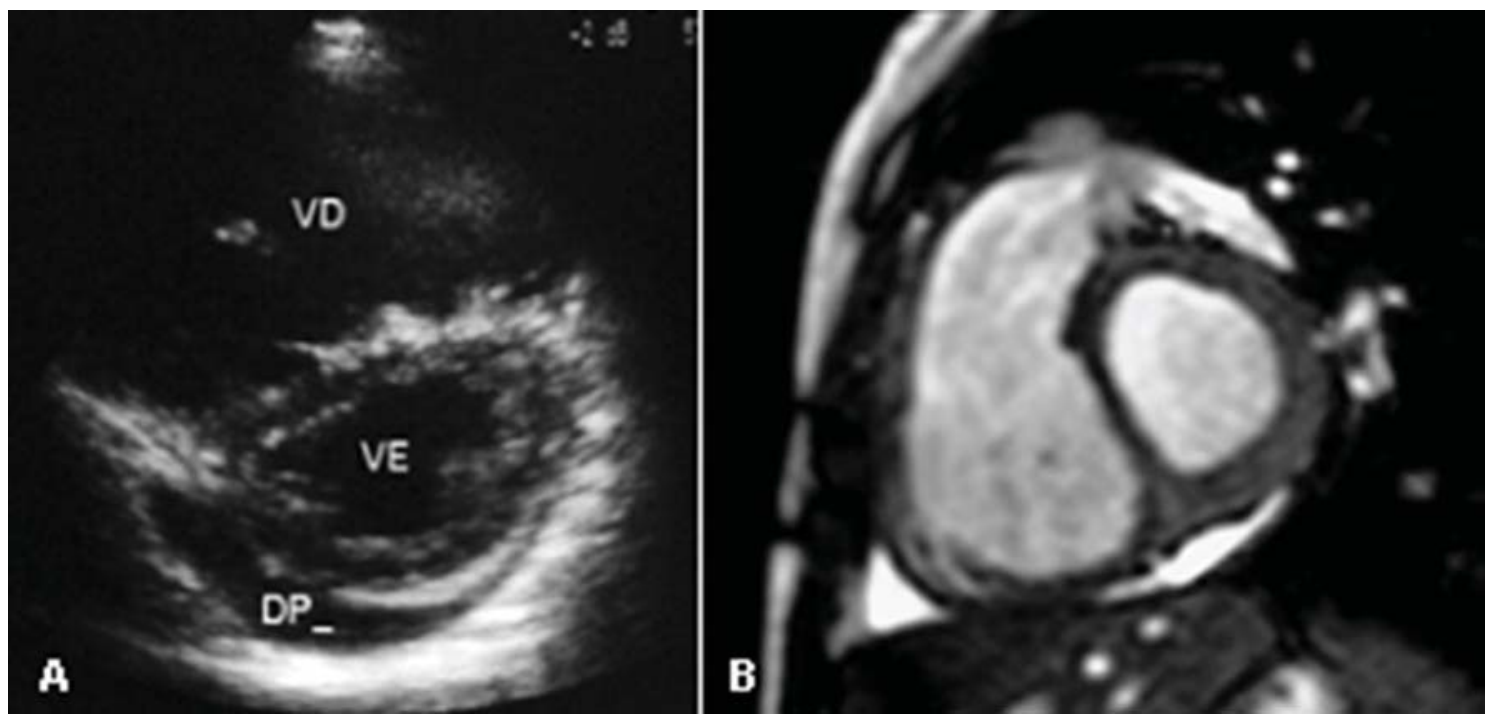

FIGURA 1

A) Ecocardiograma mostrando aumento de ventrículo direito e derrame pericárdico (caso 3). B) Ressonância magnética visualizando ventrículo direito com aumento de sua cavidade, hipertrofia importante e rechaço do septo interatrial para o ventrículo esquerdo (caso 5).

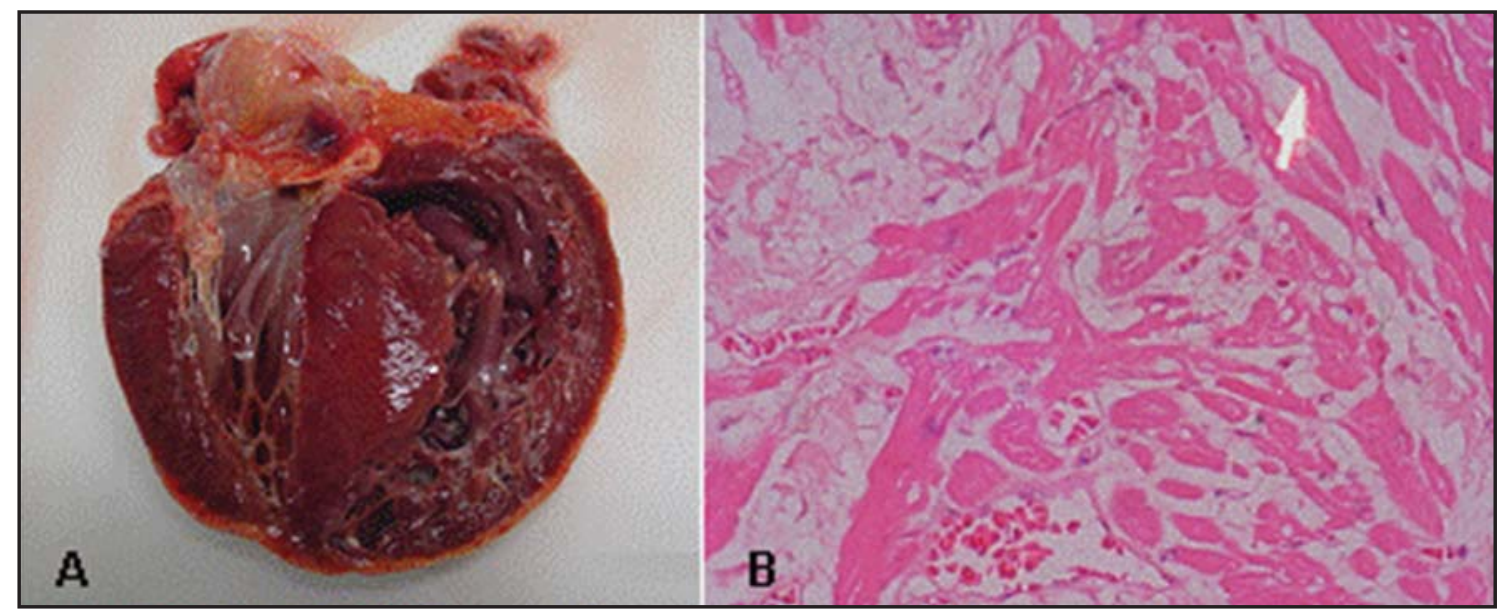

FIGURA 2

A) Hipertrofia da parede do VD e septo com dilatação da câmara ventricular. B) Hipertrofia de fibras miocárdicas do VD com desarranjo mio-sinsicial com fibrose intersticial importante (caso 2). (H-E 100x)

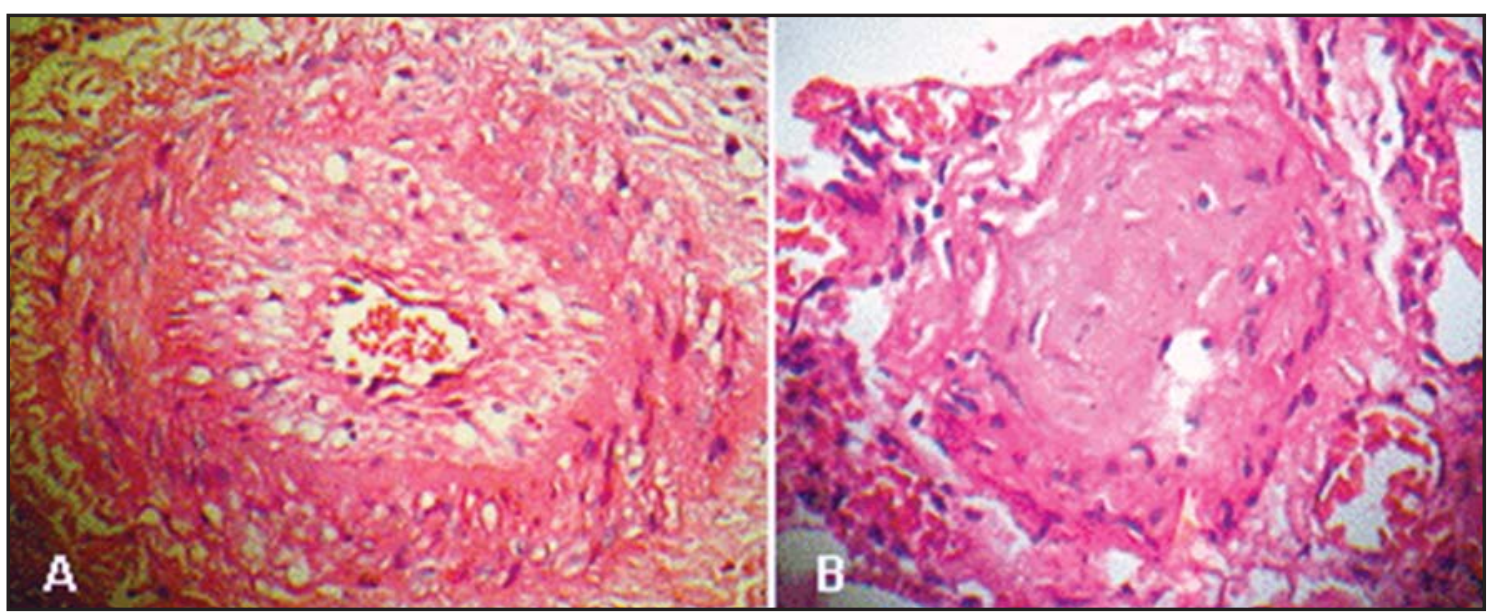

FIGURA 3

A) Artéria pulmonar distal com hipertrofia de suas paredes e obstrução quase completa de seu lúmem. B) Oclusão total da artéria pulmonar distal, com neovascularização adventicial (caso 2) (H-E 100x) 
em seus ramos mais próximos. Ao exame histológico observou-se, em vasos distais do pulmão, hipertrofia da camada íntima e média com oclusão subtotal da luz vascular, por vezes oclusão completa associada a neovascularização adventicial. 0 coração apresentou sgnificativa hipertrofia de suas fibras associada a desarranjo miosinsicial com fibrose intersticial.

\section{DISCUSSÃO}

0 primeiro caso de HAP associado ao HIV foi descrito em 1987 por Kim e Factor em um paciente hemofílico que também apresentava glomerulonefrite membranoproliferativa ${ }^{16}$. Posteriormente, diversos casos têm sido descritos em pacientes sem outro fator além da infecção pelo HIV para explicar a presença de hipertensão pulmonar ${ }^{11}$. Em recente estudo, a sobrevida média em três anos de pacientes com HAP associado ao HIV em classe funcional III e IV da NYHA foi de $28 \%$ enquanto em classe funcional I e II foi de $84 \%{ }^{26}$.

Atualmente, são muito comuns manifestações cardiovasculares não infecciosas da SIDA, como cardiomiopatia dilatada, derrame pericárdico, endocardite trombótica não bacteriana, acelerada aterosclerose e hipertensão pulmonar, todos devido a maior e melhor sobrevida assim como a profilaxia de germes oportunistas e controle da doença. Com a introdução da TARV, tem-se observado melhor controle da infecção pelo HIV podendo diminuir o risco de desenvolvimento da HAP nestes pacientes. Nos casos apresentados todos estavam em TARV.

O mecanismo de desenvolvimento da HAP em pacientes com HIV permanece desconhecido. Postula-se que o vírus poderia atuar através da liberação e ativação de mediadores como citoquinas ${ }^{14}$, fatores de crescimento ${ }^{14}$ e em particular a endotelina- ${ }^{7}$. A atuação direta do vírus no tecido pulmonar não está comprovada, devido a ausência do DNA viral nas células endoteliais pulmonares ${ }^{1422}$. Tem sido descrita maior prevalência de HLA-DR6 e HLA-DR52, em pacientes infectados pelo HIV com HAP ${ }^{24}$, porém, houve ausência da mutação BMPR2 (bone morphogenetic protein type 2) em 30 pacientes $^{26}$.

A hipertensão arterial pulmonar é uma síndrome resultando de uma redução do fluxo através da circulação pulmonar devido ao aumento da resistência vascular pulmonar e evoluindo para insuficiência ventricular direita e morte. Hoje admitida como uma doença de causa multifatorial (origem genética, molecular e celular), apresentando uma intensa remodelação vascular. É considerada hoje como uma síndrome clínica, caracterizada por sustentada elevação da pressão arterial pulmonar (PAP) e resistência vascular pulmonar (RVP) sendo definida hemodinamicamente como pressão média de artéria pulmonar (PmAP) acima de $25 \mathrm{mmHg}$ em repouso e $30 \mathrm{mmHg}$ no exercício, com pressão capilar pulmonar de $15 \mathrm{mmHg}$ ou menos, para excluir doenças cardíacas no lado esquerdo do coraçã 09 . A histopatologia da HAP idiopática é semelhante aquela HAP associada ao HIV (Figuras 2 e 3). Na doença vascular primária, a histopalogia é classificada em arteriopatia pulmonar primária (arteriopatia trombótica, hipertrofia medial, com fibrose da íntima, arteriopatia plexiforme), doença veno-oclusiva pulmonar e hemangiomatose capilar ${ }^{29}$.
Estudo em 85 macacos infectados com um retrovírus homólogo ao HIV, mostram que 19 deles desenvolveram arteriopatia similar à encontrada em pacientes com HAP associado ao HIV, mas a presença de vírus ou do DNA viral em células endoteliais não foi demonstrado ${ }^{3}$. Inflamação perivascular tem sido detectada em casos de HAP com lesões plexiformes, sugerindo que mediadores inflamatórios e fatores de crescimento podem representar fator importante no desenvolvimento da hipertensão pulmonar ${ }^{34}$. Tem sido demonstrado que proteínas do HIV ativam células endoteliais e liberam diferentes mediadores celulares como fator de necrose tumoral, endotelina-1, fator de crescimento e interleucina- $6^{72}$.

0 diagnóstico de hipertensão pulmonar em pacientes com HIV positivo, requer alto grau de suspeita clínica, pois os sinais e sintomas não são especícificos. A dispnéia é o sintoma principal em pacientes com HAP, porém, em pacientes com HIV deve-se excluir uma causa infecciosa, principalmente de origem respiratória. Todos os pacientes relatados apresentavam dispnéia e fadiga como principal sintoma, e entre outros, dor precordial, síncope e astenia (Tabela 3). Quatro dos cinco pacientes apresentaram CF III e IV de acordo com NYHA, e em todos, com galope ventricular direito, ao exame, demonstrando que, no momento do diagnóstico, estes pacientes se encontravam em avançado estágio clínico da HAP, com insuficiência ventricular direita. 0 tempo médio entre os sintomas, para estabelecer o diagnóstico de hipertensão pulmonar, em nossos pacientes com HIV foi de 4,8 meses, onde em revisão recente, foi de seis meses ${ }^{21}$, enquanto em pacientes com HAP idiopática o tempo médio tem sido de 2,5 anos $^{30}$.

A radiografia de tórax identificou alterações na grande maioria dos casos com dilatação do tronco da artéria pulmonar e cardiomegalia. 0 eletrocardiograma demonstra eixo desviado para direita e sinais de hipertrofia ventricular direita. 0 teste de caminhada de seis minutos (TC6m) é um importante instrumento para evolução da gravidade da doença, avaliação do efeito do tratamento e tem forte valor prognóstico refletindo assim a função ventricular direita ${ }^{82335}$. Em nossos pacientes, a média do TC6m foi de 104 metros, isto é, com prognóstico bastante reservado.

Os peptídeos natriuréticos (PN) são neuro-hormônios cuja concentração aumenta com sobrecarga de volume e pressão em pacientes com insuficiência cardíaca, principalmente o peptídeo natriurético cerebral terminal (NT-proBNP) ${ }^{5}$. Atualmente, o NT-proBNP tem sido empregado como marcador sérico para diagnóstico e acompanhamento terapêutico de HAP de etiologia idiopática ${ }^{25}$, associada ao tromboembolismo pulmonar agudo ${ }^{17}$. Níveis elevados têm sido associados com pior prognóstico em pacientes com insuficiência cardíaca crônica ${ }^{2}$. Níveis de NT-proBNP se correlacionam positivamente com as classes funcionais da NYHA e com parâmetros hemodinâmicos do ventrículo direito, incluindo pressão média da artéria pulmonar, resistência arterial pulmonar média e mortalidade ${ }^{25} 20$. A média de 3752ng/mL do NT-proBNP, em nosso estudo, demonstrou o grau de gravidade da descompensação ventricular direita em nossos pacientes, e os dois vivos são os que apresentam menores níveis de NT-proBNP.

A ressonância magnética de coração e pulmão é o melhor método para avaliação do ventrículo direito e medidas de 
fluxo pulmonar com grande acurácia e reprodutibilidade ${ }^{28}$. Pacientes com HAP podem apresentar reduzida fração de ejeção do ventrículo direito, com conseqüente comprometimento na capacidade funcional, tornando-se importante a identificação deste grupo ${ }^{18}$. 0 ecocardiograma é de grande importância para diagnóstico de HAP e contribui em orientar a necessidade de realização do cateterismo direito para sua confirmação diagnóstica. É também útil para descartar patologias outras como valvopatias e miocardiopatias.

0 estudo hemodinâmico por cateterização cadíaca direita e esquerda é o método padrão-ouro para diagnosticar e quantificar a HAP. Por meio de suas variáveis hemodinâmicas, podem ser identificados pacientes com pior prognóstico ${ }^{18}$. 0 grupo de pacientes apresentados neste trabalho apresentava elevadas pressões médias de átrio direito, artéria pulmonar e baixo índice cardíaco, sendo estas três principais variáveis para sobrevida e prognóstico em HAP, refletindo a história natural da falência ventricular direita ${ }^{6}$.

A terapêutica de eleição de pacientes com HAP, associada ao HIV, é desconhecida, e não tem sido muito clara se comparada à outras formas de HAP. Nenhum estudo determinou, até o momento, qual a droga de escolha para a HAP com HIV. Muitos pacientes obtêm melhora sintomática com diuréticos, digital e oxigênio $0^{4}$. Anticoagulação oral tem sido usada ainda que sua eficácia seja incerta ${ }^{114}$. A terapêutica com anticoagulante oral pode ser contraindicada devido a frequiente redução das plaquetas, dificuldade de aderência e potenciais interações com medicamentos antiretrovirais?

Em um estudo não controlado com seis pacientes, com HAP grave associada ao HIV, em uso de prostaciclina (epoprostenol), este foi efetivo em melhorar a classe funcional e hemodinâmica em 12 a 47 meses $^{1}$. Recentemente, melhoras clínicas e hemodinâmicas têm sido mostradas com o uso de bosentana em uma série de 16 pacientes com HAP associada ao $\mathrm{HIV}^{32}$. Estudos não controlados sugeriram que estes pacientes respondam favoravelmente à combinação de TARV, associada a epoprostenol e ou bosentana. 0 tratamento antiretroviral é frequentemente utilizado nestes pacientes, e alguns autores recomendam iniciar o tratamento em todos os pacientes independente da carga viral ou contagem de células CD4. Em um estudo em pacientes com HIV e HAP, tratados com TARV, houve diminuição significativa da pressão média de átrio direito em relação ao grupo não tratado $0^{27}$.

Em síntese, a hipertensão arterial pulmonar associada ao SIDA é uma doença grave onde metade dos pacientes falece em um período de seguimento de 8 meses, após o diagnóstico ${ }^{21}$. 0 HIV é reconhecidamente um fator de risco independente para o desenvolvimento de $\mathrm{HAP}^{21}$. Pacientes infectados com HIV, que desenvolvem sintomas cardiopulmonares, principalmente dispnéia inexplicada, devem ser investigados para a possibilidade de hipertensão pulmonar, porém o "screening" de rotina para HAP em pacientes com HIV, não é recomendado, devido a baixa prevalência da doença ${ }^{19}$. Estes pacientes podem responder favoravelmente à terapêutica combinada com TARV associada a agentes vasodilatadores pulmonares, prostaciclina, bosentana ou inibidores da fosfodiesterase 5 (sildenafil) ${ }^{13}$, melhorando 0 seu prognóstico.

\section{REFERÊNCIAS}

1. Aguilar RV, Farber HW. Epoprostenol (prostacyclin) therapy in HIV-associated pulmonary hypertension. American Journal Respiratory Critical Care Medicine 162:1846-1850, 2000.

2. Burger MR, Burger AJ. BNP in decompensated heart failure: diagnostic, prognostic and therapeutic potential. Current Opinion Investigation Drugs 2:929-935, 2001

3. Chalifoux LV, Simon MA, Pauley DR, MackeyJJ, Wyand MC, Ringler DJ. Arteriopathy in macaques infected with simian immunodeficiency virus. Laboratory Investigation 67:338-349, 1992

4. Conraads VM, Colebunders RL, Boshoff C. Primary pulmonary hypertension in a patient with HIV infection. Acta Cardiologica 53:367-369, 1998.

5. Cowie MR, Struthers AD, Wood DA, Coast AJ, Thompson SG, Poole-Wilson PA, Sutton GC. Value of natriuretic peptide assessment of patients with possible new heart failure in primary care. Lancet 350:1349-1353, 1997.

6. D'Alonzo GE, Barst RJ, Ayres SM, Bergofsky EH, Brundage GH, Detre KM, Survival in patients with primary pulmonary hypertension: results from a national prospective registry. Annals Internal Medicine 115:343-349, 1991.

7. Ehrenreich H, Reickmann P, Sinowatz F, Weih KA, Arthur LO, Goebel FD, Burd PR, Coligan JE, Clouse KA. Potent stimulation of monocytic endothelin-1 production by HIV-1 glycoprotein 120. Journal Immunology 150:4601-4609, 1993.

8. Galié N, Menes A, Branzi A. The new clinical trial on pharmacological treatment in pulmonary arterial hypertension. European Respiratory Journal 20:1037-1049, 2002

9. Galiè N, Torbicki A, Barst R, Dartevelle P, Haworth S, Higenbottam T, Oischewski H, Peacock A, Pietra G, Rubin LJ, Simonneau G. Guidelines on Diagnosis and Treatment of Pulmonary Arterial Hypertension of the European Society of Cardiology. European Heart Journal 25:2243-2278, 2004.

10. Goldman L, Hashimoto B, Cook EF. Comparative reproducibility and validity of systes for assessing cardiovascular functionalclass: advantages of a new specific activity scale. Circulation 64:1227-1234, 1981.

11. Himelman RB, Dohrmann M, Goodman P, Schiller NB, Starksen NF, Warnock M, Cheitlin MD. Severe pulmonary hypertension and cor pulmonale in the acquired immunodeficiency syndrome. American Journal Cardiology 64:1396-1399, 1989.

12. Hofman FM, Wright AD, Dohadwala MM, Wong-Staal F, Walker SM. Exogenous tat protein activates human endothelial cells. Blood 82:2774-2780, 1993.

13. Hoper MM, Galié N, Simonneau G, Rubin LJ. New treatments for pulmonary arterial hypertension. American Journal Respiratory Critical Care Medcine 165:1209-1216, 2002

14. Humbert M, Monti G, Fartoukh M, Magnan A, Brenot F, Rain B, Capron F, Galanaud P, Duroux P, Simonneau G, Emilie D. Platele derived growth factor expression in primary pulmonary hypertension: comparison of HIV seropositive and HIV seronegative patients. European Respiratory Journal 11:554-559, 1998.

15. Humbert M, Nunes $\mathrm{H}$, Sitbon 0 . Risk factores for pulmonary arterial hypertension. Clinical Chest Medicine 22:459-475, 2001.

16. Kim KK, Factor SM. Membranoproliferative glomerulonephritis and plexogenic pulmonary arteriopathy in a homosexual man with acquired immunodeficiency syndrome. Human Pathology 18:1293-1296, 1987.

17. Kucher N, Printzen G, Goldhaber SZ. Prognostic role of brain natriuretic paptide in acute pulmonary embolism. Circulation 107:2545-2547, 2003.

18. Loureiro R, Mendes AA, Bandeira PA, Cartaxo HQ, Sá DT. Oral sildenafil improves functional status and cardiopulmonary hemodynamic in patients with severe pulmonary hypertension secondary to chronic pulmonary schistosomiais: a cardiac magnetic resonance study. Circulation 110 (supp III): 572, 2004.

19. McLaughlin VV, Archer SL, Badesch DB, Barst RJ, Farber HW, Lindner JR, Mathier MA, McGoon MD, Park MH, Rosenson RS, Rubin LJ, Tapson VF, Varga J. ACC/AHA 2009 Expert Consensus Document on Pulmonary Hypertension. Journal of the American Colege of Cardiology 53:1573-1619, 2009.

20. MendesAA. NT-proBNP em pacientes com hipertensão pulmonar esquistossomótica: correlação clínico-hemodinâmica. Dissertação mestrado, Universidade de Pernambuco, Recife, PE, 2008. 
21. Metha NJ, Khan IA, Metha RN, Sepkowitz DA. HIV-Related pulmonary hypertension. Analytic review of 131 cases. Chest 118:1133-1141, 2000.

22. Mette AS, Palevsky HI, Pietra GG, Williams TM, Bruder E, Prestipino AJ, Patrick AM, Wirth JA. Primary pulmonary hypertension in association with human immunodeficiency virus infection. A possible viral etiology for some forms of hypertensive pulmonary arteriopathy. American Review Respiratory Disease 145:1196-1200, 1992.

23. Miaymoto S, Nagaya N, Satoh T, Kyotani S, Sakamaki F, Fujita M, Nakanishi N, Miyyatake K. Clinical correlates and prognostic significance of six-minute walk test in patients with primary pulmonary hypertension: comparison with cardiopulmonary exercise testing. American Journal Respiratory Critical Care Medicine 161:487-492, 2000.

24. Morse JH, Barst RJ, Itescu S, Flaster ER, Sinha G, Zhang Y, Fotino M. Primary pulmonary hypertension in HIV infection. An outcome determined by particular HLA class II alleles. American Journal Respiratory Critical Care Medicine 153:1299-1301, 1996

25. Nagaya N, Nishikimi I, Uematsu M, Satoh T, Kyotani S, Sakamaki F, Kakishita M, Fukushima K, Okano Y, Nakanishi N, Miyatake K, Kangaa K. Plasma brain natriuretic peptide as a prognostic indicator in patients with primary pulmonary hypertesion. Circulation 102:865-870, 2000.

26. Nunes H, Humbert M, Sitbon O, Morse JH, Deng Z, Knowles JA, Gall CL, Parent F, Garcia G, Hervé P, Barst RJ, Simonneau G. Prognostic factors for survival in human immunodeficiency virus associated pulmonary arterial hypertension. American Journal Respiratory Critical Care Medicine 167:1433-1439,2003.

27. Opravil M, Pechére M, Speich R, Joller-Jemelka HI, Jenni R, Russi EW, Hirshel B, Luthy R. HIV-associated primary pulmonary hypertension. A case control study. Swiss HIV cohort study. American Journal Respiratory Critical Care Medicine 155:990-995, 1997.
28. Pattynama PM, De Ross A, Van Der Wall EE, Van Voorthuisen AE. Evaluation of cardiac function with magnetic resonance imaging. American Heart Journal 128:595-607, 1994

29. Pietra GG, Edwards WD, Kay JM, Rich S, Kernis J, Schloo B, Ayres SM, Bergofsky EH, Brundage BH, Detre KM. Histopathology of primary pulmonary hypertension. Circulation 80:1198-1206, 1989.

30. Rich S, Dantzker DR, Ayres SM, Bergofsky EH, Brundage BH, Detre KM, Fishman AP, Goldring RM, Groves BM, Koerner SK, Levy PC, Reid LM, Vreim CE, Willians GW. Primary pulmonary hypertension. A national prospective study. Annals Internal Medicine 107:216-223, 1987.

31. Rubin LJ, Primary pulmonary hypertension. New England Journal of Medicine 336:111-117, 1997.

32. Sitbon O, Gressin V, Speich R, Macdonald OS, Opravil M, Cooper DA, Fourme T, Humbert M, Delfraissy JF, Simonneau G. Bosentan for the treatment of human imunodeficiency vírus-associated pulmonary arterial hypertension. American Journal Respiratory Critical Care Medicine 170:1212-1217, 2004.

33. Sitbon 0, Lascoux-Combe C, Delfraissy JF, Yeni PG, Raffi F, Zuttere DD, Gressin V, Clerson P, Sereni D, Simonneau G. Prevalence of HIV-related Pulmonary Arterial Hypertension in the Current Antiretroviral Therapy Era. American Journal Respiratory Critical Care Medicine 177:108-113, 2008.

34. Tuder RM, Groves B, Badesch DB, Voelkel NF. Exuberant endothelial cell growth and element of inflammation are present in plexiform lesions of pulmonary hypertension. American Journal Pathology 144:275-285, 1994.

35. Wensel R, Opitz CF, Anker SD, Winkler J, Hoffken G, Kleber FX, Sharma R, Hummel M, Hetzer R, Ewert R. Assessment of survival in patients with primary pulmonary hypertension: importance of cadiopulmonary exercise testing. Circulation 106:319-324, 2002. 